\title{
A Lagrangian Approach for the Optimal Placement of Wireless Relay Nodes in Wireless Local Area Networks
}

\author{
Aaron So and Ben Liang \\ Department of Electrical and Computer Engineering, \\ University of Toronto, Toronto, Ontario, Canada M5S 3G4 \\ \{aaronso, liang\}@ecomm.utoronto.ca
}

\begin{abstract}
The throughput capacity of WLANs can be improved by a carefully designed relay infrastructure. In this work, we propose an optimization formulation based on Lagrangian relaxation and a subgradient algorithm to compute the best placement of a fixed number of relay nodes (RNs) in a WLAN. We apply this optimization framework to a multi-rate WLAN based on the IEEE $802.11 \mathrm{~g}$ standard under Rayleigh fading. We then study the expected throughput capacity of a WLAN with relay infrastructure and investigate how the optimal placement of RNs is affected by the number of RNs, path-loss characteristics, and the traffic pattern. Our numerical results show that, in some network scenarios, more than $120 \%$ performance gain can be achieved when RNs are strategically installed in the network. Furthermore, we also show that for a wide range of system parameters, optimally placed RNs can significantly increase the network throughput capacity over random placement.
\end{abstract}

Keywords: WLAN, immobile relays, throughput capacity, optimal placement.

\section{Introduction}

Wireless Local Area Networks (WLANs), which provide low-cost wireless broadband data access for mobile Internet users, are expected to create a plethora of business opportunities. Currently, the most commonly implemented WLANs in North America are based on the IEEE $802.11 \mathrm{~b} / \mathrm{g}$ standards, which are capable of supporting bit rates up to $11 \mathrm{Mbps}$ and $54 \mathrm{Mbps}$ respectively in the $2.4 \mathrm{GHz}$ spectrum. As the number of hotspot users proliferates and the demand from wireless Internet users increases, new strategies have to be employed to increase the throughput of future WLANs.

The multi-rate capability of modern WLAN equipments and a relay infrastructure can work synergically to improve the throughput capacity of a WLAN. In a WLAN with relay infrastructure, the source can either transmit its data to the destination directly, or relay its data via a relay node $(\mathrm{RN})$. If a circuitous route can result in a higher bit rate than the direct route, the source should use the relay node to relay its data.

In this study, we investigate the optimal placement of RNs such that the throughput capacity of a WLAN can be maximized. Toward this end, we propose an analysis and optimization framework that exploits the multi-rate capability of the WLAN physical layer. Our main contributions are the following: 
- Present a tractable discretized re-formulation of the problem of optimal RN placement, which allow us to restrict the locations of the RNs.

- Solve the discrete version of the problem by computing an upper bound and a lower bound of the solution that converge toward each other, through Lagrangian relaxation and subgradient algorithm.

- Investigate the expected throughput capacity of a WLAN with relay infrastructure and how the optimal placement of RNs is affected by the number of RNs, path-loss characteristics, and traffic pattern.

The rest of this paper is organized as follows. In Section 2 we review the related work in multihop wireless networks. In Section 3, we describe the relaying architecture and define the network throughput capacity. In Section 4 we cast the general RN placement optimization problem and re-formulate it to a tractable discrete problem. We then show how this problem can be solved by Lagrangian relaxation and a subgradient algorithm. In Section 5, we present a model for IEEE 802.11g multi-rate WLAN under Rayleigh fading. In Section 6, we discuss the convergence time of the proposed optimization algorithm and show effect of different system parameters on the strategic placement of the RNs. Finally, concluding remarks are given in Section 7

\section{Related Work}

There has been much research in relaying and routing through mobile nodes. Inspired by recent advances in ad hoc networking [1], the concept of using peer mobile hosts to relay data has been explored in the context of cellular networks [2]. In a more recent work, the problem of joint routing, link scheduling and power control in such multihop networks has been investigated [3]. Moreover, issues about frequency assignment and frequency recycling in such multihop networks have been addressed in [4]. In the context of multirate WLAN, [5] and [6] have shown that by using other mobile hosts to perform relaying, the performance of the network can be improved under DCF and PCF respectively.

However, the concept of using immobile relay nodes to relay traffic, which is what we consider in this paper, has received less attention. The $i$ Car architecture [7] is one such example for the cellular environment. Immobile relay nodes have several advantages when compared with mobile relay nodes. First, because of their sedentariness, it is reasonable to assume that they have access to power supply. Consequently, energy is not a constraint. Second, the fixed relay nodes can be optimally configured to maximize their beneficial effects. The problem of fixed relay placement to maximize WLAN capacity was first studied in [8]. In [9], we proposed an efficient extension point placement algorithm aiming at improving the network layer throughput of a rectilineal network, using a divide-and-conquer searching algorithm. In this work, we explore the utilization of relay infrastructure in a discrete multi-rate WLAN, and analytically derive the optimal placement of relay nodes in such WLANs in a general network environment.

\section{Relaying Architecture and Design Objectives}

The system under consideration is analogous to a Basic Service Set (BSS) of an IEEE 802.11 WLAN. In this network configuration, there is an AP which is connected to the 
wired network, and this access point provides wireless coverage to a local area. In a network with no RN, MHs located within this coverage area directly communicate with this AP. In a network with RNs, mobile hosts located at different locations are associated with either the AP or a suitable RN. If an MH is associated with an RN, this $\mathrm{MH}$ will treat the selected RN as an AP and only communicate with the RN, so that all packets between the AP and the $\mathrm{MH}$ are relayed by the RN.

The AP communicates with each $\mathrm{MH}$ (possibly through an $\mathrm{RN}$ ) in its coverage area in a round robin fashion, thus dividing the time axis into time-varying packet transaction cycles. In each cycle, the AP transmits a downlink packet to the chosen $\mathrm{MH}$, and the $\mathrm{MH}$ transmits a uplink packet to the AP. In this study, we assume the lengths of the uplink and downlink packets may be unequal but are fixed, and the AP always has a packet to send to each active $\mathrm{MH}$ and vice versa.

We assume the transmission schedule for all transmitters is decided perfectly by the AP, and model the system as a single-channel fully-connected network. In other words, at any given time, only one transmitter is allowed to transmit, so that no packet collision is experienced at a receiver.

Let $x$ be the total number of bits of an uplink and a downlink packet combined. Let $T_{i}$ represents the packet transaction time of an AP-MH pair in the $i^{t h}$ cycle. By the Law of Large Numbers, the throughput capacity of the network is defined as

$$
C=\lim _{n \rightarrow \infty} \frac{n x}{\sum_{i=1}^{n} T_{i}}=\frac{x}{E\left[T_{i}\right]} .
$$

Therefore, in order to maximize the throughput capacity of the network, we need to minimize $E\left[T_{i}\right]$. Thus, the design objective of our system is to minimize the expected time that an AP-MH pair completes a single downlink-uplink packet exchange, which we call the packet transaction time in this paper.

We further define the packet transmission time, $T(l, P, x)$, as the expected time for a transmitter to send an $x$-bit packet to a receiver, where $l$ is the distance between the transmitter and receiver, $P$ is the reference power of the transmitter, and $x$ is the size of the packet to be transmitted. Next, we first propose an optimization method for the placement of RNs for a generic function $T(l, P, x)$, which can be obtained from theoretical models or by regression models based on site-survey results. In Section 5 , we present a case study for $T(l, P, x)$ based on the IEEE $802.11 \mathrm{~g}$ physical layer specifications with large-scale propagation path loss and Rayleigh fading.

\section{Relay Node Placement Optimization}

In this section, we first provide an analytical framework to derive the expected packet transaction time with respect to different RN placements. This analytical framework is then used to cast the RN placement problem as an optimization problem. We show that the resulting optimization can be converted to a form similar to the $p$-median problem [10] with an additional constraint. Finally, we present an efficient solution based on Lagrangian relaxation and a subgradient optimization algorithm. 


\subsection{RN Relaying}

In the elemental scenario, we consider a single $\mathrm{MH}$ and one $\mathrm{RN}$, on a plane where the $\mathrm{AP}$ is at the origin, as shown in Fig. 1] If the MH does not use the RN, the expected packet transaction time is

$$
T_{\text {norn }}(l)=T\left(l, P_{a}, x_{d}\right)+T\left(l, P_{m}, x_{u}\right) .
$$

If the $\mathrm{MH}$ uses the $\mathrm{RN}$ to relay its packet, the expected packet transaction time is

$$
T_{r n}(l, d, \theta, \varphi)=T\left(d, P_{a}, x_{d}\right)+T\left(\eta, P_{r}, x_{d}\right)+T\left(d, P_{r}, x_{u}\right)+T\left(\eta, P_{m}, x_{u}\right),
$$

where $\eta=\sqrt{l^{2}+d^{2}-2 l d \cos |\theta-\varphi|}$.

By using an $\mathrm{RN}$, the same data packet has to be transmitted twice. As a result, the RN may or may not be beneficial to an $\mathrm{MH}$. An MH will use an $\mathrm{RN}$ to facilitate its communication with the AP only if such usage result in a smaller expected packet transaction time, i.e., if $T_{r n}(l, d, \theta, \varphi)<T_{n o r n}(l)$.

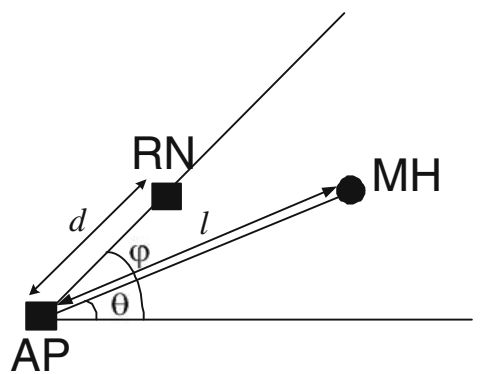

- $x_{d}=$ downlink packet length (bits).

- $x_{u}=$ uplink packet length (bits)

$-x=x_{d}+x_{u}$.

- $\beta=\frac{x_{d}}{x_{d}+x_{u}}=$ downlink proportion.

- $P_{a}=$ reference power of AP.

- $P_{r}=$ reference power of $\mathrm{RN}$.

- $P_{m}=$ reference power of $\mathrm{MH}$.

Fig. 1. Single user scenario with one relay node

\subsection{Throughput Capacity Maximization with Multiple RNs}

We assume that a fixed number, $N$, where $N>1$, of relay nodes are available for an AP in the WLAN system. Fig. 2 shows a simple example, where an AP serves the outdoor area of a campus. There are $3 \mathrm{RNs}$ available and they are placed around the AP. The coverage area, which can be in any shape, is fitted inside a circle with radius $L$ and centered at the AP. A vector, $\underline{d}$, is used to represent the displacement of RNs with respect to the AP, and a vector $\varphi$ is used to represent the angle between a predefined reference base line with respect to the radial line which each $\mathrm{RN}$ resides. Thus, we have

$$
\underline{d}=\left[d_{1}, d_{2}, \ldots, d_{N}\right]^{T}, \quad \underline{\varphi}=\left[\varphi_{1}, \varphi_{2}, \ldots, \varphi_{N}\right]^{T},
$$

where $0<d_{i} \leq L, \forall i$ and $0 \leq \varphi_{1} \leq \varphi_{2} \leq \cdots \leq \varphi_{N} \leq 2 \pi$. Moreover, the locations of RNs may be restricted due to the geographical topology. Let the set of locations where an RN can be installed be $S$. For example, in Fig. 2 , $S$ may be a subset of the perimeters of the buildings.

The MHs are distributed in the coverage area of the network with the probability density function $f(l, \theta)$. An MH may either communicate with the AP directly or select 


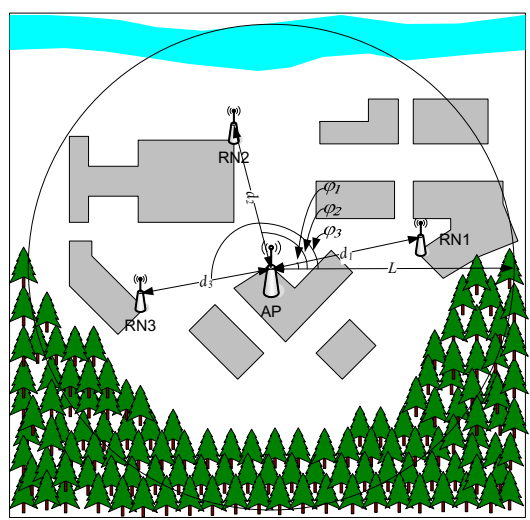

Fig. 2. Multi-user two-dimensional WLAN with multiple RNs

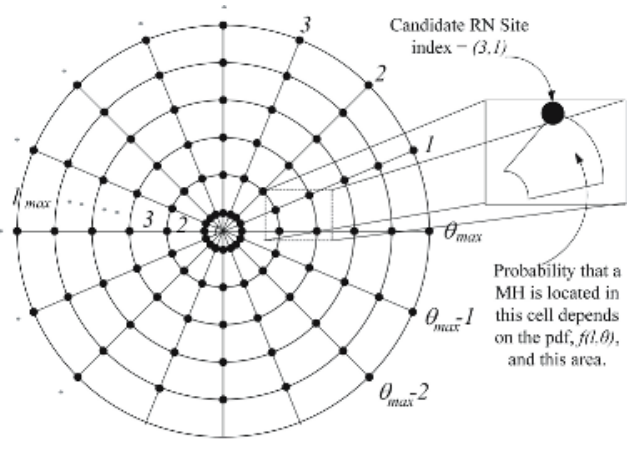

Fig. 3. Discretization of the network

the most suitable RN. Therefore, for a particular RN placement, $\left(d_{i}, \varphi_{i}\right) \in S$ for $i=$ $1, \ldots, N$, the expected packet transaction time of the network can be computed as

$$
\overline{T_{r n}(\underline{d}, \underline{\varphi})}=\int_{0}^{2 \pi} \int_{0+\epsilon}^{L} l f(l, \theta) \min \left[T_{n o r n}(l), \min _{1 \leq k \leq N} T_{r n}\left(l, d_{k}, \theta, \varphi_{k}\right)\right] d l d \theta,
$$

where $\epsilon>0$ is small. Using (5), we have the following optimization problem:

$$
\begin{aligned}
\text { Objective: } & \min _{\underline{d}, \underline{\varphi}} \overline{T_{r n}(\underline{d}, \underline{\varphi})} \\
\text { s.t. } & 0<d_{i} \leq L, \quad \forall i \\
& 0 \leq \varphi_{1} \leq \varphi_{2} \leq \cdots \leq \varphi_{N} \leq 2 \pi \\
& \left(d_{i}, \varphi_{i}\right) \in S \quad \forall i .
\end{aligned}
$$

Clearly, this problem is difficult to solve directly. However, in reality, there is no need to determine the RN placement with sub-meter granularity. Hence, we can calculate the approximate value of (5) by discretizing the network into a large but finite number of areas, where a mobile host is located at each area with a certain probability. Then, the integral in (5) can be interpreted as a Riemann sum.

\subsection{Problem Reformulation}

As shown in Fig. 3, we can divide the entire network into $\theta_{\max }$ equal-size sectors, and each sector is then divided into $l_{\max }$ equal-length cells $11 \mathrm{~A}$ mobile host is located at the corner of each cell with a certain probability, and the area of the cell represents the area that this mobile host occupies. Moreover, the corner of each cell also represents a candidate site of the RN set. Each $\mathrm{MH}$ or RN candidate site can be uniquely identified by its radial line number and its cell number. For example, the selected site in Fig. 3 lies on the third cell of the first radial line. Thus, this site is indexed by $(3,1)$. For notation

\footnotetext{
${ }^{1}$ In our numerical analysis, the network is divided into approximate 100 thousand cells.
} 
purpose, we use $(i, j)$ to describe the location of an $\mathrm{MH}$, while we use $(\delta, \tau)$ to represent the location of an RN candidate site. We define the following notations:

$$
\begin{aligned}
\Delta \theta & =\frac{2 \pi}{\theta_{\max }}, \quad \Delta l=\frac{L}{l_{\max }}, \quad \underline{\delta}=\left(\delta_{1}, \ldots, \delta_{N}\right)^{T}, \quad \underline{\tau}=\left(\tau_{1}, \ldots, \tau_{N}\right)^{T}, \\
d_{k} & \approx \delta_{k} \Delta l, \quad \text { for } 1 \leq k \leq N, \text { and } \delta_{k} \in \mathbf{Z}^{+}, \text {and } 1 \leq \delta_{k} \leq l_{\max }, \\
\varphi_{k} & \approx \tau_{k} \Delta \theta, \quad \text { for } 1 \leq k \leq N, \text { and } \tau_{k} \in \mathbf{Z}^{+}, \text {and } 1 \leq \tau_{k} \leq \theta_{\max }, \\
h_{(a, b)} & =\int_{(b-1) \Delta \theta}^{b \Delta \theta} \int_{(a-1) \Delta l}^{a \Delta l} l f(l, \theta) d l d \theta, a \in\left[1, l_{\max }\right], b \in\left[1, \theta_{\max }\right], \\
S^{\prime} & =M(S),
\end{aligned}
$$

where $M(\cdot)$ is the mapping of a set from the continuous space to the discrete space. Furthermore, for notation simplification, we let $T_{r n}^{\Delta l, \Delta \theta}(i, \delta, j, \tau)=T_{r n}(i \Delta l, \delta \Delta l$, $j \Delta \theta, \tau \Delta \theta)$, and $T_{n o r n}^{\Delta l}(i)=T_{n o r n}(i \Delta l)$. Then, (5) can be approximated as

$$
\begin{aligned}
\overline{T_{r n}(\underline{d}, \underline{\varphi})} & =\int_{0}^{2 \pi} \int_{0+\epsilon}^{L} l f(l, \theta) \min \left[T_{n o r n}(l), \min _{1 \leq k \leq N} T_{r n}\left(l, d_{k}, \theta, \varphi_{k}\right)\right] d l d \theta \\
& \approx \sum_{i=1}^{l_{\max }} \sum_{j=1}^{\theta_{\max }} \min \left[T_{n o r n}^{\Delta l}(i), \min _{1 \leq k \leq N} T_{r n}^{\Delta l, \Delta \theta}\left(i, \delta_{k}, j, \tau_{k}\right)\right] h_{(i, j)} \\
& =\overline{T_{r n}(\underline{\delta}, \underline{\tau})}
\end{aligned}
$$

To facilitate the minimization of $\overline{T_{r n}(\underline{\delta}, \underline{\tau})}$, we define two sets of decision variables, $\mathbf{X}$ and $\mathbf{Y} . X_{(\delta, \tau)}=1$ if an $\mathrm{RN}$ is placed in position $(\delta, \tau)$; otherwise, $X_{(\delta, \tau)}=0$. Moreover, $Y_{(i, j),(\delta, \tau)}=1$ if the $\mathrm{MH}(i, j)$ is served by $\mathrm{RN}(\delta, \tau)$; otherwise, $Y_{(i, j),(\delta, \tau)}=0$.

Note that $X_{(0,0)}=1$ because the access point is always present. Moreover, since $X_{(0,0)}=1$, we have $Y_{(i, j),(0,0)}=1$ only if the $\mathrm{MH}$ at $(i, j)$ is served directly by the AP. Thus, (6) can be reformulated as

$$
\begin{aligned}
& \min _{\mathbf{X}, \mathbf{Y}}: \sum_{i=1}^{l_{\max }} \sum_{j=1}^{\theta_{\max }}\left[h_{(i, j)} T_{n o r n}^{\Delta l}(i) Y_{(i, j),(0,0)}+\sum_{\delta=1}^{l_{\max }} \sum_{\tau=1}^{\theta_{\max }} h_{(i, j)} T_{r n}^{\Delta l, \Delta \theta}(i, \delta, j, \tau) Y_{(i, j),(\delta, \tau)}\right] \\
& \text { s.t. } Y_{(i, j),(0,0)}+\sum_{\delta=1}^{l_{\max }} \sum_{\tau=1}^{\theta_{\max }} Y_{(i, j),(\delta, \tau)}=1 \quad \forall(i, j) \\
& \sum_{\delta=1}^{l_{\max }} \sum_{\tau=1}^{\theta_{\max }} X_{(\delta, \tau)}=N \\
& X_{(0,0)}=1 \\
& Y_{(i, j),(\delta, \tau)}-X_{(\delta, \tau)} \leq 0 \\
& \forall(i, j),(\delta, \tau) \\
& X_{(\delta, \tau)}=0 \\
& \forall(\delta, \tau) \notin S^{\prime}
\end{aligned}
$$

Objective (8) minimizes the expected minimum packet transaction time of all MHs in the network. Constraint (9) requires each $\mathrm{MH}$ to be assigned to exactly one $\mathrm{RN}$ or the AP. Constraint (10) states that exactly $N$ RNs are to be located. Constraint (11) 
states that the AP is always present. Constraint (12) requires that the $\mathrm{MH}$ at $(i, j)$ can be assigned to an $\mathrm{RN}$ at $(\delta, \tau)$ only if an $\mathrm{RN}$ is installed at location $(\delta, \tau)$. Constraint (13) required that the RNs are not located in the infeasible sites.

\subsection{An Optimization-Based Lagrangian Relaxation Iterative Algorithm}

Lagrangian relaxation with subgradient optimization can be used to provide approximate solution to many NP-hard problems efficiently. Thus, noting the distinctive characteristics of our RN placement formulation, we propose to solve our optimization problem in (8) as follows.

Step 1: Setting up. We relax constraint (9) and obtain (14), where $\lambda_{(i, j)}$ are the Lagrange multipliers. In our numerical analysis below, all $\lambda_{(i, j)}$ values are initialized to 5000 .

$$
\begin{aligned}
\max _{\lambda} \min _{\mathbf{X}, \mathbf{Y}} & \sum_{i=1}^{l_{\max }} \sum_{j=1}^{\theta_{\max }} \lambda_{(i, j)}\left[1-Y_{(i, j),(0,0)}-\sum_{\delta=1}^{l_{\max }} \sum_{\tau=1}^{\theta_{\max }} Y_{(i, j),(\delta, \tau)}\right]+ \\
& \sum_{i=1}^{l_{\max }} \sum_{j=1}^{\theta_{\max }}\left[h_{(i, j)} T_{n o r n}^{\Delta l}(i) Y_{(i, j),(0,0)}+\sum_{\delta=1}^{l_{\max }} \sum_{\tau=1}^{\theta_{\max }} h_{(i, j)} T_{r n}^{\Delta l, \Delta \theta}(i, \delta, j, \tau) Y_{(i, j),(\delta, \tau)}\right] \\
= & \sum_{i=1}^{l_{\max }} \sum_{j=1}^{\theta_{\max }} \lambda_{(i, j)}+\sum_{i=1}^{l_{\max }} \sum_{j=1}^{\max }\left[\left(h_{(i, j)} T_{n o r n}^{\Delta l}(i)-\lambda_{(i, j)}\right) Y_{(i, j),(0,0)}+\right. \\
& \left.\sum_{\delta=1}^{l_{\max }} \sum_{\tau=1}^{\theta_{\max }}\left(h_{(i, j)} T_{r n}^{\Delta l, \Delta \theta}(i, \delta, j, \tau)-\lambda_{(i, j)}\right) Y_{(i, j),(\delta, \tau)}\right]
\end{aligned}
$$

s.t. [10), (11), (12), 13) are satisfied .

Step 2: Solving the simplified problem. For fixed values of the Lagrange multipliers, we want to minimize the objective function (14). Since the values of $\lambda_{(i, j)}$ are fixed, the first term in the objective function, which is just the sum of all Lagrangian multipliers, is a constant. To minimize (14), we begin by computing the value of

$$
V_{(\delta, \tau)}=\sum_{i=1}^{l_{\max }} \sum_{j=1}^{\theta_{\max }} \min \left(0,\left[h_{(i, j)} T_{r n}^{\Delta l, \Delta \theta}(i, \delta, j, \tau)-\lambda_{(i, j)}\right]\right) \quad \forall(\delta, \tau) \in S^{\prime}
$$

We then find the $N$ smallest values of $V_{(\delta, \tau)}$ and set the corresponding values of $X_{(\delta, \tau)}=$ 1 and all other values of $X_{(\delta, \tau)}=0$. We then $\operatorname{set} Y_{(i, j)(\delta, \tau)}=1$ if $h_{(i, j)} T_{r n}^{\Delta l, \Delta \theta}(i, \delta, j, \tau)-$ $\lambda_{(i, j)}<0$ and $X_{(\delta, \tau)}=1$. Moreover, since $X_{(0,0)}=1$, we set $Y_{(i, j)(0,0)}=1$ if $h_{(i, j)} T_{\text {norn }}^{\Delta l}(i)-\lambda_{(i, j)}<0$. All other $Y$ 's are set to zero.

Step 3: Updating the lower and upper bounds. For each iteration of this process, an upper bound and lower bound of the original objective function (8) need to be determined. From Step 2, $N$ RN candidate sites are selected. The expected minimum packet transaction time with this particular placement can be calculated by using (7). This value 
is an upper bound of (8). The lower bound for the current iteration is simply the objective function (14) with the values of $\mathbf{X}$ and $\mathbf{Y}$ found in Step 2 [11].

Step 4: Modifying the Lagrange multipliers. The Lagrange multipliers are revised using a standard subgradient optimization procedure [11]. At the $n^{\text {th }}$ iteration of the Lagrangian procedure, we first compute the step size by

$$
t^{n}=\frac{A^{n}\left(U B-\mathcal{L}^{n}\right)}{\sum_{i=1}^{l_{\max }} \sum_{j=1}^{\theta_{\max }}\left[Y_{(i, j),(0,0)}^{n}+\sum_{\delta=1}^{l_{\max }} \sum_{\tau=1}^{\theta_{\max }} Y_{(i, j),(\delta, \tau)}^{n}-1\right]^{2}},
$$

where $U B$ and $\mathcal{L}^{n}$ are the upper and lower bounds found from Step 3, $Y_{(i, j),(\delta, \tau)}^{n}$ is the optimal value of $Y_{(i, j),(\delta, \tau)}$ at the $n^{\text {th }}$ iteration, and $A^{n}$ is a constant updated as follows. We begin with $A^{1} \leq 2$ an arbitrary small positive number. At each iteration, the value of $A^{n}$ is halved if $\mathcal{L}^{n}$ has not increased in $c_{A}$ consecutive iterations. In our numerical analysis, we use $A^{1}=2$ and $c_{A}=4$. Then, the Lagrangian multipliers are updated by

$$
\lambda_{(i, j)}^{n+1}=\max \left[0, \lambda_{(i, j)}^{n}-t^{n}\left(Y_{(i, j),(0,0)}^{n}+\sum_{\delta=1}^{l_{\max }} \sum_{\tau=1}^{\theta_{\max }} Y_{(i, j),(\delta, \tau)}^{n}-1\right)\right] .
$$

Step 5: Iteration and termination. The algorithm terminates when any one of the following conditions is true:

1. A predefined number of iterations are completed.

2. The upper bound equals or is close enough to the lower bound.

3. $A^{n}$ is small, such that the changes in $\lambda_{(\delta, \tau)}$ becomes too small. Such small changes are not likely to help solve the problem.

Otherwise, we repeat from Step 2.

\section{IEEE 802.11 Model and Packet Transmission Time}

In this section, as a sample case study, we derive the expected packet transmission time based on the IEEE $802.11 \mathrm{~g}$ bit rate model. Suppose there are $M$ data rates, denoted $r_{1}, r_{2}, \ldots, r_{M}$, supported by the physical layer. Reliable communication by using rate $r_{m}$ can be realized only if the signal strength at the receiver is above a certain threshold, say $\eta_{m}$. Consequently, for the set of $M$ data rates, there is a set of $M$ thresholds, $\eta_{1}, \ldots, \eta_{M}$. We further define $\eta_{0}=0$ and $\eta_{M+1}=\infty 2$

We study the case where the following large-scale propagation model is applicable: $P_{2}=\frac{P_{1}}{d_{2}^{\alpha}}$, where $P_{1}$ is the reference signal power measured at one meter away from the transmitter, $P_{2}$ is the signal power measured at $d_{2}$ meters away from the transmitter, and $\alpha$ is a positive constant representing the path loss roll off factor. The reference power $P_{1}$ can be obtained via field measurement or calculated using the free space path loss formula in [12].

${ }^{2}$ In IEEE $802.11 \mathrm{~g}$, there are 11 different bit rates, and the minimal threshold for each bit rate is specified by the standard. 
In addition to large scale propagation, multipath fading may have a prominent effect on reliable communication. Under Rayleigh fading, the instantaneous power, $\gamma$, is exponentially distributed with the probability density function $p(\gamma)=\frac{1}{P_{r}} e^{\frac{-\gamma}{P_{r}}}$, where $P_{r}$ is the average power of $\gamma$. Consequently, the probability that a transmitter with reference power $P$ can transmit at rate $r_{m}$, to a receiver at distance $l$, where $l>1$, is $p\left(r_{m}, l, P\right)=\int_{\eta_{m}}^{\eta_{m+1}} \frac{l^{\alpha}}{P} e^{\frac{-\gamma l^{\alpha}}{P}} d \gamma$, where $m=1,2, \ldots, M$. Furthermore, in some instances, the receiver can be located in a deep-fade area, i.e., is experiencing bad channel condition. The probability of these instances, where the transmitter cannot transmit in any data rate, is $p_{f}(l, P)=\int_{\eta_{0}}^{\eta_{1}} \frac{l^{\alpha}}{P} e^{\frac{-\gamma l^{\alpha}}{P}} d \gamma$, while the probability that the transmitter can transmit successfully is $p_{s}(l, P)=1-p_{f}(l, P)=\sum_{m=1}^{M} p\left(r_{m}, l, P\right)$.

In this discrete-rate model with fading, the transmitter needs a small channel probing time, denoted $T_{\text {prob }}$, to test the channel and decide the transmission rate before the actual data transmission can take place. If the transmitter determines that the channel condition does not allow it to transmit at any rate, it will give up its transmit opportunity, and prob the channel again later. The wasted channel probing time adds to the total packet transmission time.

Let $T_{g}(l, P, x)$ be a random variable that represents the packet transmission time of an $x$-bit packet, and let $S$ and $F$ be the events of "good" and "bad" channel states respectively. The expected value of this packet transmission time is

$$
\begin{aligned}
& E\left[T_{g}(l, P, x)\right] \\
& =E\left[T_{g}(l, P, x) \mid F\right] p_{f}(l, P)+E\left[T_{g}(l, P, x) \mid S\right] p_{s}(l, P) \\
& =\left(E\left[T_{g}(l, P, x)\right]+T_{\text {prob }}\right) p_{f}(l, P)+\left(\sum_{m=1}^{M} \frac{p\left(r_{m}, l, P\right)}{p_{s}(l, P)}\left[\frac{x}{r_{m}}+T_{\text {prob }}\right]\right) p_{s}(l, P) .
\end{aligned}
$$

Rearranging the above, we have the expected packet transmission time

$$
T(l, P, x)=E\left[T_{g}(l, P, x)\right]=\frac{T_{\text {prob }}}{p_{s}(l, P)}+\sum_{m=1}^{M} \frac{p\left(r_{m}, l, P\right)}{p_{s}(l, P)} \frac{x}{r_{m}} .
$$

\section{Numerical Analysis}

In this section, we present numerical results from the proposed optimization methods and evaluate the capacity improvement from using wireless relay nodes in an urban WLAN. Unless otherwise stated, the system parameters such as bit rate power thresholds, antenna gains, and transmitter powers are taken from the CISCO Aironet 1100 Series Access Point and mobile network interface card specifications [13]. The other system parameters are selected based on a typical urban environment.

\subsection{Convergence of Lagrangian Iteration}

In Fig. 4 we show the convergence of the algorithm in two typical network scenarios. For both scenarios, the network provides a coverage area of 400 meters in radius, and 16 


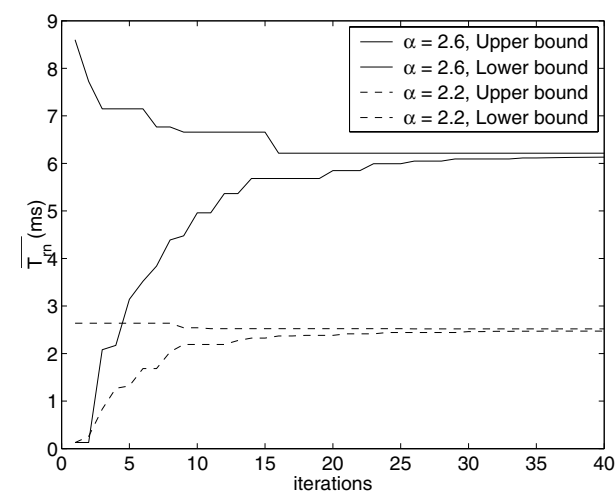

Fig. 4. Example convergence of the Lagrangian relaxation iterative algorithm

RNs are available to be placed in this network without restriction. MHs are uniformly distributed in the network coverage area. Both the AP and RN are equipped with a $10 \mathrm{dBm}$ transmitter, while the mobile hosts use a $5 \mathrm{dBm}$ transmitter. The combined length of a uplink and a downlink packet is set to $2 \mathrm{k}$ bytes, and $70 \%$ of downlink traffic is assumed. By default, the network is discretized into 100 thousand cells, corresponding to approximately 5 square meters per cell on average.

As shown in Fig. 4 for both channel roll-off factors, $\alpha=2.2$ and $\alpha=2.6$, the difference between the upper bound and the lower bound converges to less than $2 \%$ of the lower bound value in less than 40 iterations.

\subsection{Effect of System Parameters on RN Placement and Performance Gain}

In this subsection, we discuss the benefit of the strategically placed RNs with respect to different system parameters. The system that we investigated has three system parameters: roll off factor $(\alpha)$, proportion of downlink data $(\beta)$, and the number of RNs $(N)$. For each set of parameters, our analysis and optimization procedure produces an optimal placement of RNs. Moreover, as defined in (1), we can calculate the throughput capacity of the network without relay nodes, $C_{\text {norn }}$ and with relay nodes optimally placed, $C_{r n}$. Hence, $C_{n o r n}$ and $C_{r n}$ are defined as $\frac{x}{\overline{T_{n o r n}}}$ and $\frac{x}{\overline{T_{r n}\left(\underline{\left.d^{*}, \underline{\varphi^{*}}\right)}\right.}}$ respectively, where $\left(\underline{d^{*}}, \underline{\varphi^{*}}\right)$ represents the optimal RN location(s) calculated by the Lagrangian relaxation iterative algorithm. We define the performance gain of utilizing the RNs as Gain $=100 \times \frac{C_{r n}-C_{n o r n}}{C_{n o r n}}$. In the following, the relationship among the three system parameters with respect to the optimal RN placement and performance gain will be discussed. Moreover, we also compare the optimal performance gain with the performance gains resulting from random placements of $\mathrm{RNs} 3$

We study the effect of the roll off factor, $\alpha$, and the number of RNs, $N$, in Fig. 5 and Fig. 6 Except $N$ and $\alpha$, the same set of system parameters from subsection 6.1 are used. Fig. 5 and Fig. 6 show the performance gains and optimal RN placements with respect

\footnotetext{
${ }^{3}$ For each set of system parameters, 100 different random RN placements were generated, and we report the average performance gain of relaying using randomly place RNs.
} 


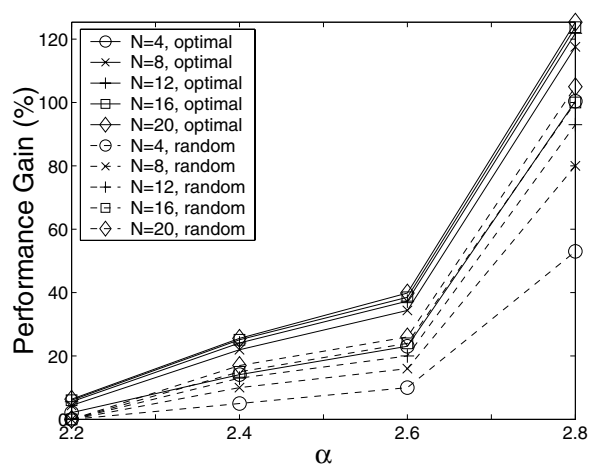

Fig. 5. Performance gain with respect to different roll-off factors and number of RNs

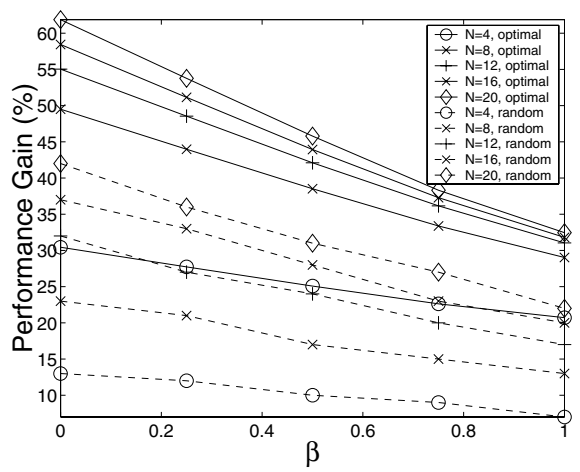

Fig. 7. Performance gain with respect to different proportion of downlink data and number of RNs

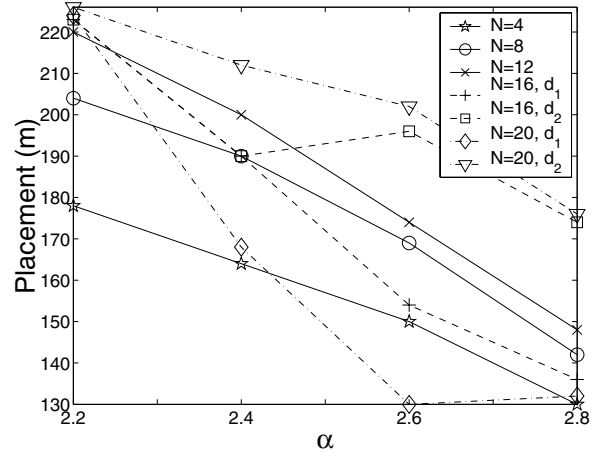

Fig. 6. Optimal placement of RNs with respect to different roll-off factors and number of RNs

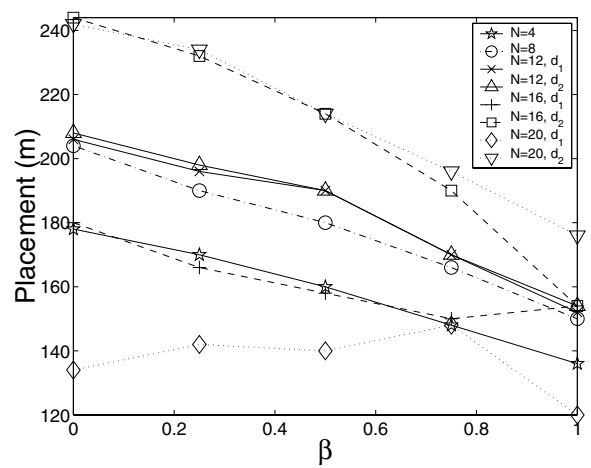

Fig. 8. Optimal placement of RNs with respect to different proportion of downlink data and number of RNs

to different roll off factors respectively. When there are 4,8 or $12 \mathrm{RNs}$, the solution calculated by the Lagrangian algorithm converges to a single-tier configuration, where the RNs are uniformly distributed around and with a displacement $d_{1}$ meters away from the AP. When there are 16 or 20 RNs available, the Lagrangian algorithm converges to a two-tier configuration, where two equal-size rings of RNs with radius $d_{1}$ and $d_{2}$ are formed surrounding the AP.

From these figures, we make three main observations. First, the performance gain is high when the pathloss roll-off factor is high. The roll off factor determines how fast the signal decays when it travels through a distance. Therefore, as the roll off factor increases, the benefits of the RNs become more significant. Second, the performance gain difference between optimal and random placement of the RNs is substantial when the number of $\mathrm{RN}$ is small to moderate. Third, in all cases, the effect of diminishing return is observed as the number of RNs increases. These observations suggest that when the number of RN is high, the marginal gain of each addition $\mathrm{RN}$ is small. 
We study the effect of the proportion of downlink data $(\beta)$ and the number of RNs $(N)$ on an urban network in Fig. 7 and Fig. 8 . The system parameters are the same as before, except the roll off factor is set to 2.6. Again, the combined length of a uplink and a downlink packet is set to $2 \mathrm{k}$ bytes. Thus, the downlink and uplink packet lengths are $2 \beta \mathrm{k}$ bytes and $2(1-\beta) \mathrm{k}$ bytes respectively.

When there are 4 or $8 \mathrm{RNs}$, the Lagrangian algorithm converges to an single-tier configuration regardless of the proportion of downlink data. When there are 12, 16 or $20 \mathrm{RNs}$, the algorithm converges to a two-tier configuration, similar to the previous subsection.

Two main observations can be seen from these figures. First, the performance gain increases as the proportion of uplink data, $(1-\beta)$, increases. This is because the MH's transmitter has less power compared with that of the AP and RNs. As the amount of data needed to be transmitted by the MH's transmitter increases, the benefit of the RN becomes more significant. Second, relaying with optimally placed RNs performs significantly better than that of random placement regardless of traffic pattern.

\section{Conclusions}

In this work, we have investigated the strategic placement of wireless relay nodes to enhance the throughput capacity of an urban wireless local area network. We have developed an analytical model for performance evaluation and RN placement optimization. We propose a Lagrangian relaxation iterative algorithm to solve a discrete version of the RN placement problem. The proposed framework can be generalized to fit different channel models, network configurations, and user behaviors. In particular, we have investigated the RN placement problem in an IEEE 802.11g multi-rate WLAN under Rayleigh fading. Using the proposed numerical analysis framework, we have showed that in most cases, by using strategically placed RNs, the network capacity can be significantly improved. Given a set of network parameters, the proposed algorithm can be used by network designers to compute the optimal placement of RNs and justify the tradeoff between additional hardware cost and system performance gain.

\section{References}

1. Haas, Z.J., Deng, J., Liang, B., Papadimitratos, P., Sajama, S.: Wireless ad hoc networks. In Proakis, J., ed.: Wiley Encyclopedia of Telecommunications. John Wiley \& Sons (2002)

2. Lin, Y., Hsu, Y.: Multihop cellular: A new architecture for wireless communications. In: Proc. of IEEE INFOCOM. (2000) 1273 - 1282

3. Cruz, R., Santhanam, A.: Optimal routing, link scheduling and power control in multihop wireless networks. In: Proc. of IEEE INFOCOM. (2003) 702-711

4. Mengesha, S., Karl, H., A.Wolisz: Capacity increase of multi-hop cellular WLANs exploiting data rate adapatation and frequency recycling. Technical report, Technical University Berlin Telecommunication Networks Group (2003)

5. Zhu, H., Cao, G.: rDCF: A relay-enabled medium access control protocol for wireless ad hoc networks. In: Proc. of IEEE INFOCOM. (2005) 12-22

6. Zhu, H., Cao, G.: On improving the performance of IEEE 802.11 with relay-enabled PCF. ACM/Kluwer Mobile Networking and Applications (MONET) 9 (2004) 423-434 
7. Wu, H., Qiao, C., De, S., Tonguz, O.: Integrated cellular and ad hoc relaying systems: iCAR. IEEE Journal on Selected Areas in Communications 19(10) (2001) 2105-2215

8. So, A., Liang, B.: Effect of relaying on capacity improvement in wireless local area networks. In: Proc. of IEEE WCNC. (2005) 1539 - 1544

9. So, A., Liang, B.: An efficient algorithm for the optimal placement of wireless extension points in rectilineal wireless local area networks. In: Proc. of International Conference on Quality of Service in Heterogeneous Wired/Wireless Networks (QShine). (2005) 25-33

10. Daskin, M.: Network and Discrete Location: Models, Algorithms and Applications. John Wiley \& Sons (1995)

11. Martin, R.K.: Large Scale Linear and Integer Programming. Kluwer Academic Publishers (1999)

12. Rappaport, T.S.: Wireless Communications: Principles and Practice. Prentice Hall (2001)

13. CISCO: Aironet 1100 series access point: Data sheet. Technical report, CISCO Systems (2003) 